\title{
The electronic structure of liquid water within density functional theory
}

\author{
David Prendergast, Jeffrey C. Grossman, and Giulia Galli \\ Lawrence Livermore National Laboratory, L-415, P.O. Box 808, Livermore, CA 94551.
}

(Dated: November 13, 2018)

\begin{abstract}
In the last decade, computational studies of liquid water have mostly concentrated on ground state properties. However recent spectroscopic measurements have been used to infer the structure of water, and the interpretation of optical and x-ray spectra requires accurate theoretical models of excited electronic states, not only of the ground state. To this end, we investigate the electronic properties of water at ambient conditions using ab initio density functional theory within the generalized gradient approximation (DFT/GGA), focussing on the unoccupied subspace of Kohn-Sham eigenstates. We generate long (250 ps) classical trajectories for large supercells, up to 256 molecules, from which uncorrelated configurations of water molecules are extracted for use in DFT/GGA calculations of the electronic structure. We find that the density of occupied states of this molecular liquid is well described with 32 molecule supercells using a single k-point $(\mathbf{k}=\mathbf{0})$ to approximate integration over the first Brillouin zone. However, the description of the density of unoccupied states (u-EDOS) is sensitive to finite size effects. Small, 32 molecule supercell calculations, using the $\Gamma$ point approximation, yield a spuriously isolated state above the Fermi level. Nevertheless, the more accurate u-EDOS of large, 256 molecule supercells may be reproduced using smaller supercells and increased k-point sampling. This indicates that the electronic structure of molecular liquids like water is relatively insensitive to the long-range disorder in the molecular structure. These results have important implications for efficiently increasing the accuracy of spectral calculations for water and other molecular liquids.
\end{abstract}

PACS numbers: 71.15.Dx,71.15.Mb,78.40.Dw,78.40.Pg

\section{INTRODUCTION}

Our understanding of the structure of water in its many phases is fundamental to research in fields as diverse as biochemistry, cellular biology, atmospheric chemistry, and planetary physics. The standard experimental approaches to the analysis of crystal structures - x-ray $\stackrel{\underline{1}}{\underline{1}}$ and neutron ${ }^{2}$ diffraction - can provide detailed information on ordered phases of ice, but only indirect, and often limited, information on amorphous ice and liquid water. Nonetheless, the results of these experiments have permitted major advances in the understanding of the properties of water in the last 30 years; in addition they made possible the development of simple classical potentials to describe water in the condensed phase, thus enabling molecular dynamics simulations to probe its dynamical properties.

Recently, more sophisticated ab initio electronic structure approaches have become available to perform simulations of water ${ }^{3} \mathrm{Ab}$ initio molecular dynamics based on density functional theory ${ }^{4.5}$ (DFT) has been extensively used to study water and solvation processes. It is not yet fully understood how accurate DFT is $\frac{6.7}{}$ - and, in particular, how accurate the various gradient corrected functionals ${ }^{8.9 .10}$ are - in describing the structural and diffusive properties of liquid water. Furthermore, the quantitative influence of the inclusion of proton quantum effects in ab initio simulations remains to be established ${ }^{11}$ However, recent results reported in the literature have shown that qualitative features of hydrogen bonding in liquid water are correctly accounted for by ab initio molecular dynamics (MD) based on DFT

The majority of $a b$ initio studies, to date, have concen- trated on structural properties of liquid water, and, only recently, have first-principles calculations of absorption spectra been carried out.13,14,15,16.17,18,19 These computations were motivated by new spectroscopic results obtained using x-ray Raman spectroscopy ${ }^{19.20 .21}$ and x-ray absorption spectroscopy ${ }^{15.17 .18 .22}$ for water and ice; and $\mathrm{x}$-ray absorption spectroscopy for liquid water jets. ${ }^{23,24}$ The experimental spectra - providing direct information on the electronic transitions from atomic core levels to excited states - have been used to infer information on the structural properties of the fluid. In particular, the number of hydrogen bonds and the details of the hydrogen bonded network in the fluid have been inferred from experiment and compared to those of ice. A standard procedure has been to use DFT in the gradient corrected approximation to compute absorption spectra of selected snapshots representing the liquid - within some approximation for the description of the core-hole interaction. Those snapshots producing spectra in agreement with experiment have then been considered to be representative of the "correct" water structure, as probed experimentally. While this may well be a viable and straightforward approach in the absence of major approximations in the theory used to compute spectra, it becomes a much more complex interpretative tool in the presence of approximations in the theory, in particular approximations regarding the core-hole interaction with the excited electron.

In order to provide a clear interpretation of measured and computed spectra, an important prerequisite is to fully understand the electronic structure of the fluid, as described within DFT. The purpose of this article is to provide a detailed description of the electronic structure 
of liquid water using Kohn-Sham density functional theory, and in particular to understand in detail the unoccupied subspace of Kohn-Sham eigenstates - especially the conduction band minimum, which will have a great influence in determining the onset of computed absorption spectra.

We shall show that the electronic density of states (EDOS) of liquid water, near the conduction band minimum, is particularly sensitive to finite size effects. We find that for small, 32 molecule supercell calculations of the DFT electronic structure of liquid water, approximating Brillouin zone integration by sampling the $\Gamma$-point only, leads to an EDOS where the lowest unoccupied state is separated from the rest of the conduction band by $\sim 1.5 \mathrm{eV}$. This is consistent with previous DFT calculations of the electronic structure of water.

In order to understand this peculiar feature of the EDOS of water, we first examine the isolated dimer and the ordered hexagonal phase of ice I $h$. The KohnSham eigenstate energies of the dimer indicate a separation in energy of the lowest unoccupied molecular orbital (LUMO) from the rest of the unoccupied states. This is apparently consistent with the separation of such states in liquid water. However, water possesses a hydrogen bonded network more similar to ice than to the isolated dimer. An examination of the band structure of the ice $\mathrm{I} h$ crystal indicates large dispersion in the conduction band states. In particular, there is a large separation $(\sim 3 \mathrm{eV})$ between the two lowest conduction bands at the $\Gamma$-point.

Inspired by these observations, we examine the convergence of the EDOS of liquid water with respect to k-point sampling. We compute the EDOS using several uncorrelated configurations of water molecules generated using molecular dynamics at ambient conditions with a classical potential. Examining the EDOS of several liquid water configurations, we find that the occupied portion is converged using just one k-point. This implies that the results of previous DFT studies of water in the electronic ground state would be unaffected by increased k-point sampling. However, for the unoccupied portion of the EDOS, the separation between the two lowest conduction bands is merely an artifact of poor k-point sampling of the Brillouin zone. We test whether the EDOS computed using a 32 molecule supercell with $8 \mathrm{k}$-points is an accurate approximation of the EDOS computed using a 256 molecule supercell with $1 \mathrm{k}$-point. Such calculations would yield identical results for a periodic crystal; however, for a disordered phase like liquid water this equivalence is destroyed. Yet, at $300 \mathrm{~K}$ the differences are small, and we find that increased $\mathrm{k}$-point sampling in smaller supercells is indeed an accurate approximation of the EDOS of much larger liquid water systems. This indicates that our converged, k-point sampled EDOS of liquid water is accurate and that the presence of an isolated LUMO of water is spurious.

Our analysis has important consequences for the calculation of spectral properties (both optical and x-ray) of pure water and materials in aqueous solvation, since the

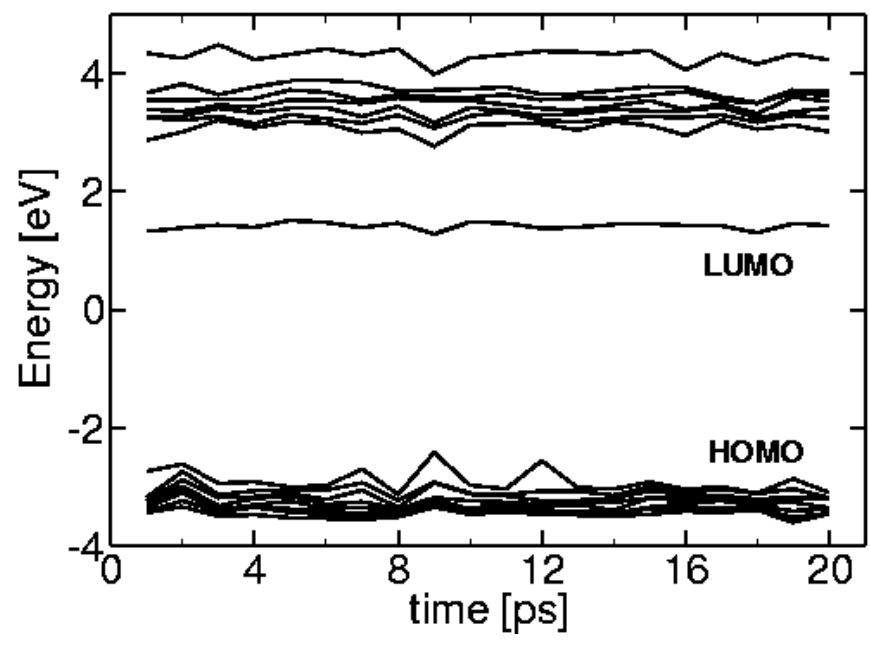

FIG. 1: Occupied and unoccupied Kohn-Sham eigenenergies in the neighborhood of the Fermi energy (HOMO), computed using DFT/PBE for sequential molecular configurations of 32 water molecules taken from a 20 ps Car-Parrinello MD simulation at $300 \mathrm{~K}$, using only the $\Gamma$-point. There exists a clear separation in energy between the LUMO and the rest of the conduction band.

accuracy of these quantities is dependent on a reliable description of the unoccupied EDOS.

The outline of our work is as follows: Before presenting our results, we discuss some possible misunderstanding of the conduction band in water, which exists in the literature (Section II ). To this end, we build some intuition on the electronic structure of liquid water based on an isolated water dimer and a crystalline phase of ice (Sections III and IV respectively). We efficiently generate a large number of molecular configurations of liquid water, for use in DFT calculations, using classical molecular dynamics, as indicated in Section $\nabla$. In Sections VI and VII we make use of these MD trajectories to illustrate that, while the $\Gamma$-point approximation is valid for Brillouin zone integration in the occupied subspace of Kohn-Sham eigenstates in liquid water, this is in fact a very poor approximation for the unoccupied states. To indicate the accuracy of the electronic structure computed with increased k-point sampling, we compare in Section VIII with the EDOS of larger supercell calculations. We outline in Section IX the implications that such improvements in the description of the electronic structure will have on calculations which make use of the unoccupied EDOS, and in Section $\mathrm{X}$ we show that our approach is quite efficient as a means of increasing the accuracy of these calculations. Finally, we give our conclusions in Section XI 


\section{PREVIOUS DESCRIPTIONS OF THE LOWER CONDUCTION BAND OF LIQUID WATER}

The earliest $a b$ initio calculations on liquid water ${ }^{25}$ reported the existence of a delocalized lowest unoccupied molecular orbital (LUMO). This LUMO comprised some molecular $\sigma^{*}$ character on the oxygens of each water molecule and a delocalized tail which extended throughout the system in the intermolecular volume, avoiding hydrogen-bonds. In addition, the reported electronic density of states (EDOS) for a supercell of 32 heavy-water molecules $\left(\mathrm{D}_{2} \mathrm{O}\right)$, calculated within DFT using the BeckePerdew gradient corrected exchange correlation functiona ${ }^{8.26 .27}$ reveals an unexpected, isolated state at the bottom of the conduction band. This is perhaps counterintuitive, as one might expect that a molecular system would possess bands of almost degenerate states arising from weak coupling of the original isolated molecular orbitals; therefore, the conduction band minimum would be the lowest in energy of a band of states produced by coupling of the LUMOs of single water molecules.

Later work ${ }^{28}$ displayed the Kohn-Sham eigenenergies of water, as a function of time during a Car-Parrinello molecular dynamics ${ }^{3}$ simulation, at densities lower than the ambient density. This showed the isolated peak above the Fermi energy of liquid water to be caused by a single Kohn-Sham eigenstate, whose separation from the rest of the conduction band grows with water density. The authors of this work infer that this "delocalized LUMO can be regarded as the precursor of the solvated electron." At these low densities they state that this LUMO has more delocalized character than those other virtual orbitals higher in energy, which possess more molecular character. Subsequent work ${ }^{29}$ simulated the occupation of this LUMO with an excess electron, performing Car-Parrinello MD at ambient conditions, leading to the formation of a localized region of charge at intervals of approximately $0.1 \mathrm{ps}$ mediated by the breaking of hydrogen bonds. The calculated optical absorption from these simulations showed good agreement with hydrated excess electon experiments 30

Since then, a large body of work on water and solvated systems has generated similar time-dependent EDOS, all exhibiting a clear separation of the LUMO of water from the rest of the conduction band. 31.32.33.34 For clarity, we report in Fig. 1 a similar set of KohnSham eigenenergies as a function of time for a CarParrinello MD simulation of 32 water molecules at ambient density $\left(\rho=1.0 \mathrm{~g} / \mathrm{cm}^{3}\right)$ and temperature $(300 \mathrm{~K})$. The Car-Parrinello trajectory was generated using the GP code $^{35}$ and the Kohn-Sham eigenenergies of the unoccupied states for the configurations taken from this trajec-

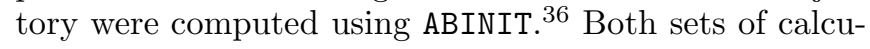
lations used DFT in the generalized gradient approximation (GGA) of Perdew, Burke and Ernzerhof (PBE) 37 These were planewave, norm-conserving pseudopotential,, 38 electronic structure calculations performed in a
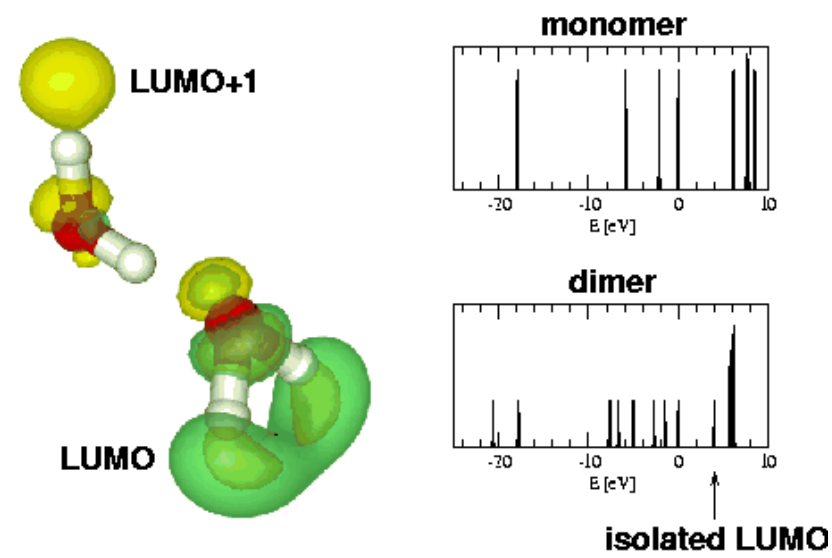

FIG. 2: The water dimer in its ground state geometry computed using DFT/PBE. Left: Isosurfaces of the probability densities of the first two unoccupied Kohn-Sham eigenstates above the Fermi energy - LUMO and LUMO+1. Water molecules are indicated using red (oxygen) and white (hydrogen) ball-and-stick models. Right: A comparison of the Kohn-Sham eigenenergies of the monomer and the dimer indicating a separation in energy between LUMO and LUMO+1. Note that the energies in both cases have been shifted to align the HOMO with zero.

supercell under periodic boundary conditions. We truncate the planewave basis using a kinetic energy cut-off of 70 Ry and the oxygen pseudopotential is non-local in the $s$ angular momentum channel. The Brillouin zone integration is approximated using a single k-point, $\mathbf{k}=\mathbf{0}$ (the $\Gamma$-point). This is a standard approximation for reasonably large supercells of insulating systems and is the same approach used in previous work, which led to EDOS of water similar to that of Fig. 1 with an isolated LUMO state.

The presence of such an isolated LUMO is not apparent from the experimentally measured optical spectrum of water $\stackrel{39.40}{ }$ which displays no noticeable peak near the onset of absorption. This, however, does not discount the presence of such a peak in the unoccupied EDOS, which could be diminished in the spectrum by reduced oscillator strengths associated with optical transitions from the top of the valence band. In the following sections we shall examine the electronic structure of the water dimer (the smallest hydrogen bonded water system) and of hexagonal ice (a fully saturated hydrogen bonded phase). From this analysis we shall develop an improved picture of the unoccupied EDOS of liquid water and show, ultimately, that there is no isolated peak in the EDOS at the bottom of the conduction band.

\section{THE WATER DIMER}

Past justifications for the presence of an isolated LUMO in the u-EDOS of liquid water emphasized comparisons with the molecular orbitals of the water dimer. 
We used DFT/PBE to compute the electronic structure of the water monomer and dimer. Note that all subsequent DFT calculations outlined in this work were performed using the PWSCF package ${ }^{41}$ and with a planewave cut-off of $85 \mathrm{Ry}$, unless stated otherwise . Two KohnSham eigenstates of the dimer, the LUMO and the KohnSham eigenstate just above it in energy $(\mathrm{LUMO}+1)$, are indicated by isosurfaces of their probability densities in Fig. 2 It is observed by comparison of the Kohn-Sham eigenenergies of the dimer and monomer that the LUMO is lower in energy than the LUMO+1 as a consequence of being localized on the dangling hydrogen bonds in this system. Similarly, in the case of liquid water, the LUMO is $\operatorname{seen}^{25}$ to possess a similar $\sigma^{*}$ component on the oxygens in the system and also a significant probability density in those regions where the hydrogen-bond network is disrupted.

\section{BAND STRUCTURE OF ICE Ih}

Liquid water is perhaps more akin to ice than a gas phase dimer. In ordered phases of ice, all hydrogen bonds are saturated (i.e., four hydrogen bonds per water molecule) and, molecular dynamics simulations of liquid water at ambient conditions indicate that the average number of hydrogen bonds per molecule is between 3 and 4, depending on the particular definition of a hydro-

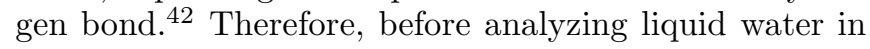
detail, we choose to analyze the electronic structure of ice.

Previous work ${ }^{43.44}$ on the band structure of cubic ice (Ic) and the density of states of hexagonal ice 45 (I $h$ ) gives no indication of peculiarity at the conduction band minimum. However, the band structures indicate significant dispersion in the bands just above the Fermi energy. Using the hexagonal unit cell of ice $\mathrm{I} h$ as pro-

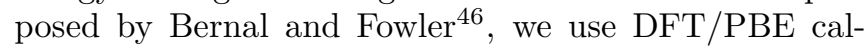
culations to generate the structural parameters within the Born-Oppenheimer approximation at a pressure and temperature of $\sim 0.1 \mathrm{MPa}$ and $0 \mathrm{~K}$, respectively. Proton disorder is not considered while we use the primitive unit cell containing 12 water molecules with all hydrogen bonds passivated (Fig. 3). This structure has a density of $1.002 \mathrm{~g} / \mathrm{cm}^{3}$, corresponding to hexagonal lattice parameters of $a=7.59 \AA$ and $c=7.18 \AA$. The use of the PBE functional is justified by previous work on ice at various pressures ${ }^{47}$ We present the band structure in Fig. 4. The self-consistent electronic charge density is computed using $8 \mathrm{k}$-points in the Brillouin zone and all eigenenergies in the band structures computed non-selfconsistently using this charge density and its associated Kohn-Sham potential. Our band structure is similar to that reported by Hahn et al.,$\frac{48}{4}$ where they employ a cubic cell with some proton disorder, and we use the primitive hexagonal cell with no proton disorder.

We note immediately that the large degree of dispersion in the unoccupied subspace leads to a separation of
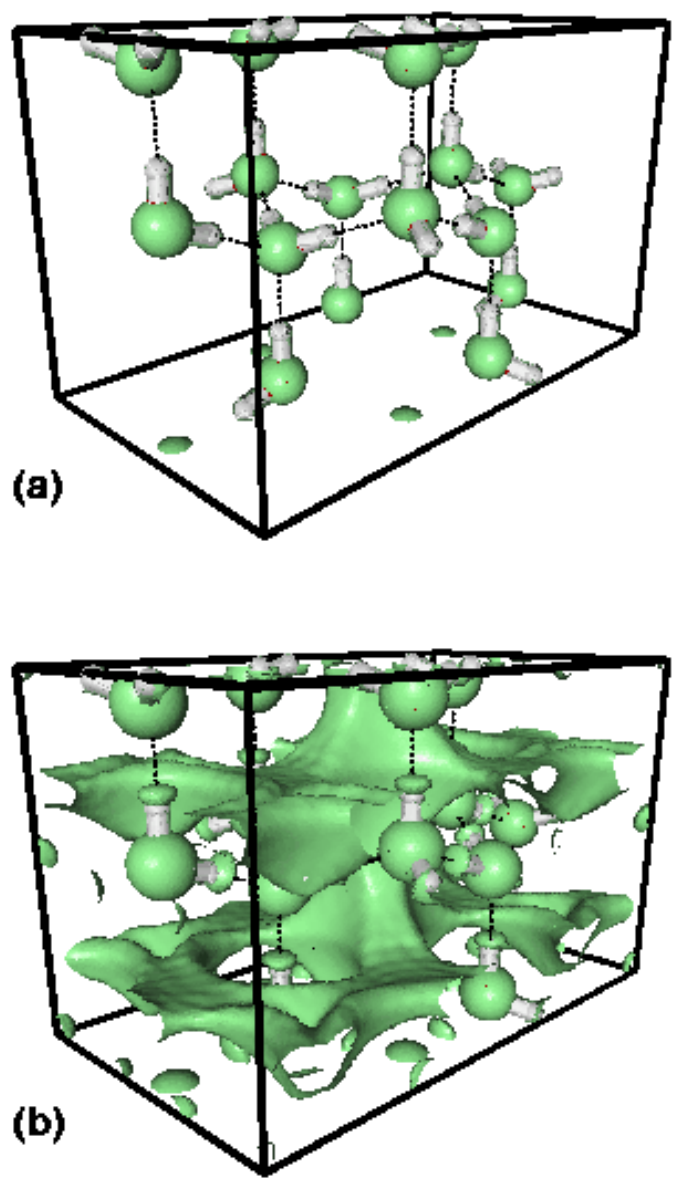

FIG. 3: The ice I $h$ structure with isosurfaces of the probability density (green) of the Kohn-Sham eigenstate just above the Fermi energy at the $\Gamma$-point: (a) the oxygen $\sigma^{*}$ component; and (b) the delocalized, hydrogen-bond-avoiding component, corresponding, respectively, to $\sim 5 \%$ and $\sim 30 \%$ of the integrated density of the state. Water molecules are indicated using red (oxygen) and white (hydrogen) ball-and-stick models. Hydrogen bonds indicated as dashed lines.

the lowest conduction bands similar to that between the LUMO and LUMO +1 in previous calculations for water. This separation is particularly large at the $\Gamma$-point. It is clear that considering only the band structure at the $\Gamma$-point in Fig. 4 would lead to a similar EDOS as that outlined for liquid water in Fig. 1 Also, the Kohn-Sham eigenstate at the bottom of the conduction band is delocalized, with $\sigma^{*}$ character on the oxygen atoms and an avoidance of hydrogen bonds, similar to the LUMO in previous water calculations. Furthermore, the separation between the lowest two conduction bands at the $\Gamma$-point is $3.0 \mathrm{eV}$. This large energy is consistent with the trends indicated by Boero et al. for the variation of this separation with density 28 The discrepancy between this large separation in ice $\mathrm{I} h$ and that of $\sim 1.5 \mathrm{eV}$ in ambient liquid water (which has a similar density) is likely due to 


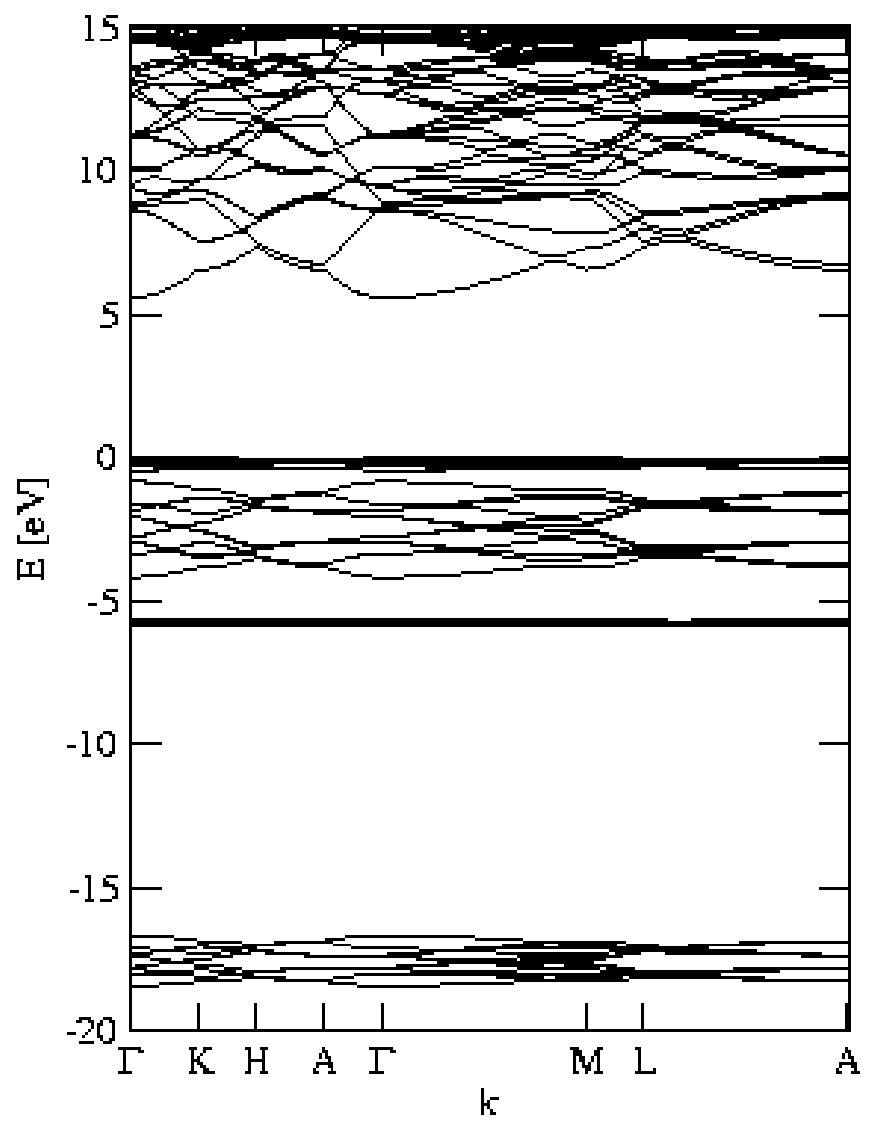

FIG. 4: The band structure of ice Ih computed using DFT/PBE for the 12 molecule unit cell, with structural parameters optimized to reduce the pressure to $\sim 0.1 \mathrm{MPa}$. The electronic charge density is determined using $8 \mathrm{k}$-points in the first Brillouin zone. (see Fig. [3] and text).

the saturation of all hydrogen bonds in the former, leading to a more delocalized state and consequently more dispersion at the $\Gamma$-point.

\section{CLASSICAL TIP4P TRAJECTORIES FOR LIQUID WATER}

In an attempt to facilitate the efficient reproduction of our results, we use configurations of water molecules generated using a classical potential. This removes the computational expense of generating such configurations using $a b$ initio $\mathrm{MD}$, and also provides configurations with structures in closer agreement with experiment, at least for such measures as the radial distribution functions and diffusion coefficient. Recent, careful, ab initio DFT/GGA MD simulations have been shown to produce more structured radial distribution functions in comparison with experiment 6.7.11 We use the TIP4P four-site mode ${ }^{49}$, which has been shown recently to have a wide range of transferability for water in various condensed phases .50 We see in Fig. [5 that TIP4P approximates well the experimentally determined ${ }^{2}$ oxygen-oxygen ra-

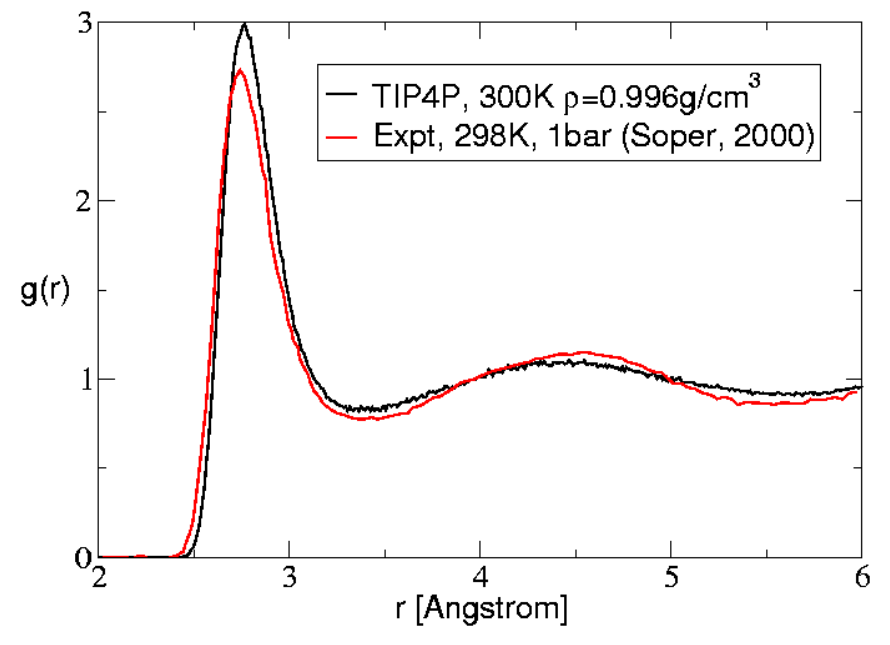

FIG. 5: The oxygen-oxygen radial distribution function of liquid water at ambient conditions determined experimentally $^{2}$ (red) and from a molecular dynamics simultion using the TIP4P classical potential.

dial distribution function of water at ambient conditions.

We used the gromacs molecular dynamics package ${ }^{51,52}$ to generate long trajectories for large supercells of water molecules in the $N V T$ ensemble. We choose the density to be $0.996 \mathrm{~g} / \mathrm{cm}^{3}$ and the temperature to be $300 \mathrm{~K}$. MD simulations for all supercell sizes reported here are begun using a box of water molecules cut from a large (2048 molecule) equilibrated sample. The molecules in this supercell are provided with a Boltzmann velocity distribution consistent with a temperature of $300 \mathrm{~K}$ and allowed to equilibrate for 50 ps. A further $200 \mathrm{ps}$ is evolved for sampling purposes. All configurations used for DFT calculations are separated by 20 ps. Previous work indicates that this separation is of the same order of magnitude as the structural correlation time. 6

\section{VI. "BAND STRUCTURE" OF LIQUID WATER}

We provide in Fig. [6 the DFT/PBE calculated "band structure" for a representative cubic supercell of 32 water molecules extracted from the equilibrated section of a TIP4P classical trajectory. We recognize that the concept of a band structure has no meaning for an aperiodic system, however the band structures of the periodic approximations to the true disordered systems provide information about the electronic structure of the liquid. Convergence of the electronic charge density with respect to k-point sampling in self-consistent calculations for this system indicated that using just the $\Gamma$-point is a valid approximation rather than attempting a full integration of the Brillouin zone. This fact is clearly evidenced by the lack of significant dispersion in the occupied subspace of this liquid water configuration. We computed the KohnSham eigenvalues at each $\mathrm{k}$-point in this band structure using the effective potential derived from the electronic 


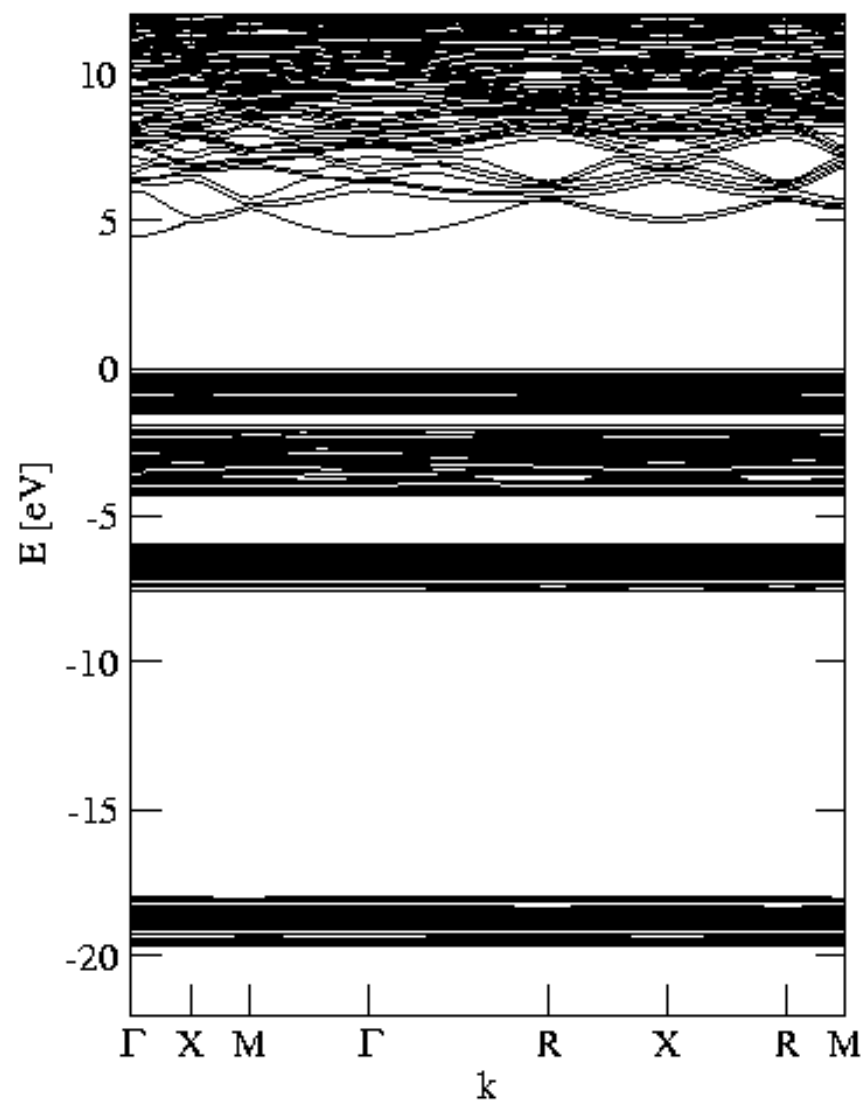

FIG. 6: Computed DFT/PBE "band structure" for one representative 32 molecule supercell of liquid water taken from a TIP4P trajectory. The electronic charge density is converged using the $\Gamma$-point only.

charge density computed using the $\Gamma$-point approximation.

Examination of the unoccupied bands in this small liquid water supercell reveals large dispersion, particularly at the $\Gamma$-point. This indicates that the $u$-EDOS of this periodic system will be sensitive to the amount of $\mathrm{k}$-point sampling employed. Clearly, using the EDOS at the $\Gamma$ point will lead to the familiar separation of states at the bottom of the conduction band as seen in Fig. 1] However, we can see now that increasing k-point sampling will add to the EDOS in the region that has traditionally separated the LUMO and LUMO+1 in $\Gamma$-point calculations of liquid water using supercells containing 32 water molecules. We examine this convergence in the next section.

\section{CONVERGENCE OF THE EDOS WITH K-POINT SAMPLING}

We take 10 uncorrelated molecular configurations of 32 water molecules of liquid water, at ambient conditions, at intervals of $20 \mathrm{ps}$ from a TIP4P trajectory. For each of these we perform DFT/PBE electronic structure calculations, increasing the k-point sampling of the first Brillouin zone. The indicated number of $\mathrm{k}$-points refers to the number of points in a uniform grid about $\mathbf{k}=\mathbf{0}$. For example, $8 \mathrm{k}$-points implies a $2 \times 2 \times 2$ grid. However, using the symmetry of our cubic supercells we reduce the actual number of k-points used in the calculation, reweighting each appropriately in the sum which approximates a complete Brillouin zone integration. In each of the configurations examined we find that $64 \mathrm{k}$ points is sufficient to converge the EDOS to the accuracy necessary for this demonstration. Note that more rapid convergence can sometimes be achieved by generating $\mathrm{k}$ point grids centred about a k-point other than $\Gamma$. We did not test such grids in this work.

The results of these convergence tests are displayed in Fig. 7 Close examination of the enlarged EDOS at $\mathrm{t}=0$ ps demonstrates that the occupied EDOS (below zero in energy) remains essentially unchanged with $\mathrm{k}$ point sampling. The only noticeable effect is some reduction in the sharpness of features when using the $\Gamma$-point with small numerical broadening ( $0.05 \mathrm{eV}$ in this case). However, we see clearly that the unoccupied EDOS is greatly modified as the k-point density is increased. The gap that exists between LUMO and LUMO+1 (from $2 \mathrm{eV}$ to $3.5 \mathrm{eV}$ ) under the $\Gamma$-point approximation is filled completely at higher k-point densities. Furthermore, the qualitative form of the EDOS beyond the conduction band minimum is completley different. Comparison with the other uncorrelated molecular configurations reveals the same behavior. In fact, as a function of time, the converged EDOS shows more variation below the Fermi energy than it does in the unoccupied subspace. These variations are likely due to particular relative orientations of water molecules or making and breaking of hydrogen bonds, but will not be investigated here.

\section{CONVERGENCE OF THE EDOS WITH SYSTEM SIZE}

For an electronic structure calculation of a periodic system, accurate Brillouin zone integration may be achieved either by (i) increasing the k-point sampling of the Brillouin zone of the primitive unit cell, or (ii) by using larger supercells comprising repeated unit cells and a minimal k-point sampling. The latter approach is clearly more expensive and where possible we would prefer the former option. However, for disordered systems, the equivalence of these two approaches no longer holds, since the system is no longer periodic. Therefore, it is, in principle, more accurate within calculations performed under periodic boundary conditions to approach the limit of the bulk, disordered phase by increasing the supercell size and using the $\Gamma$-point approximation. This is also a requirement for the analysis of long-range molecular structure in the disordered phase.

However, experience from tight-binding calculations of a variety of systems, indicates that the electronic struc- 

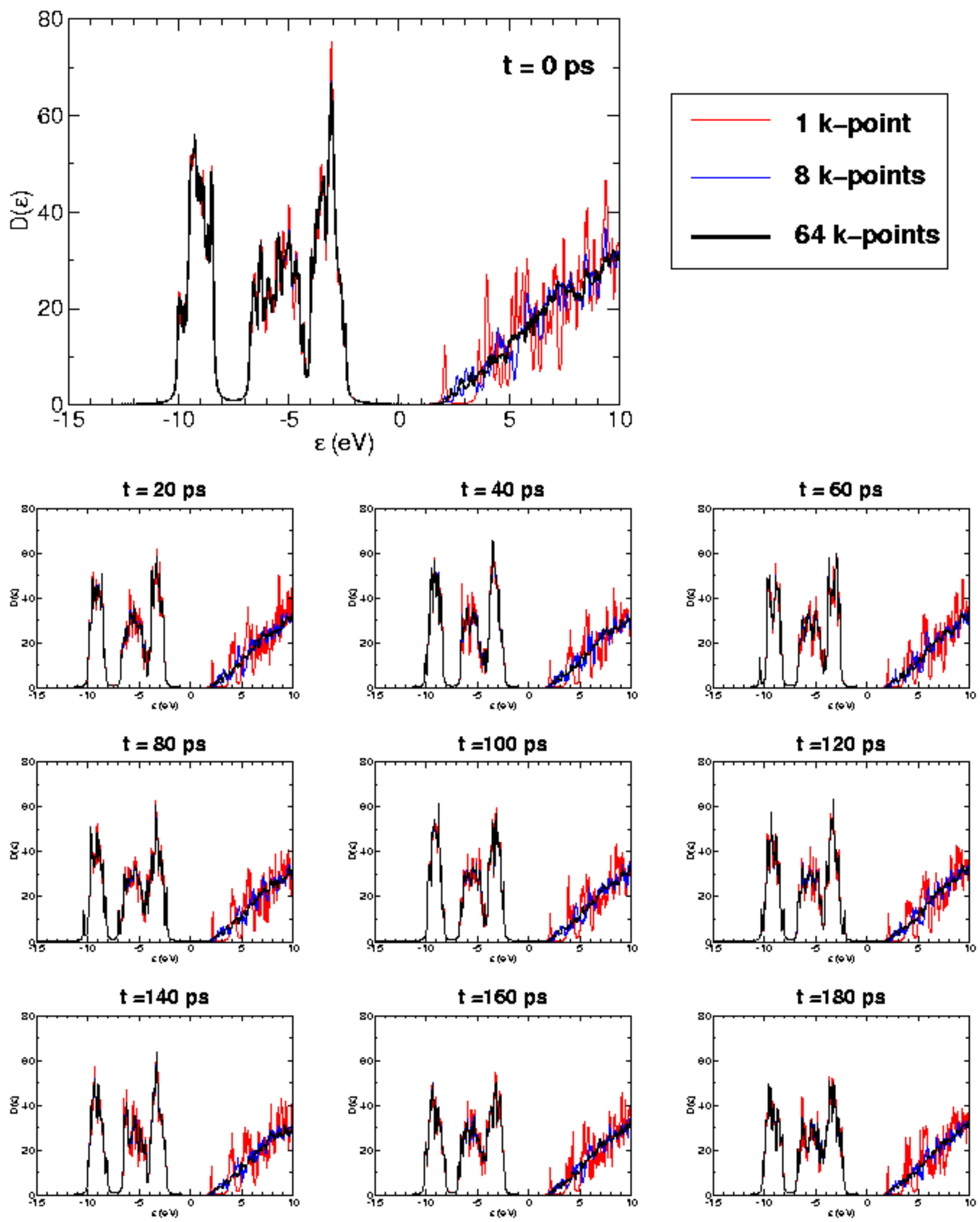

FIG. 7: Convergence of the EDOS, computed within DFT/PBE, with respect to k-point sampling for 10 uncorrelated configurations of 32 water molecules sampled every 20ps from a 200 ps TIP4P, $N V T$ molecular dynamics trajectory at T=300 K, $\rho=0.996 \mathrm{~g} / \mathrm{cm}^{3}$. The EDOS are broadened using Lorentzians with a full width at half maximum of $0.05 \mathrm{eV}$. 

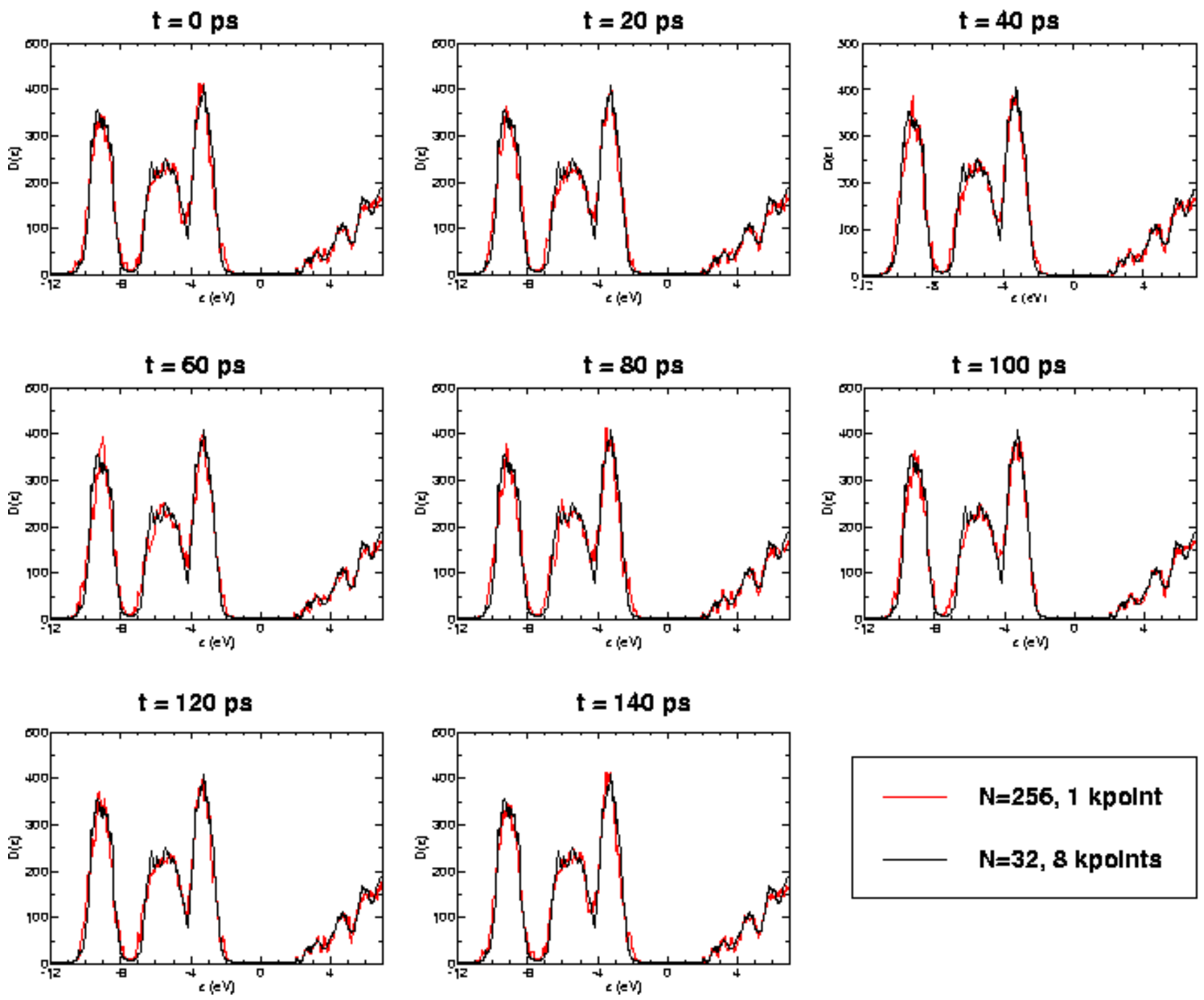

FIG. 8: Comparison of EDOS for liquid water, computed within DFT/PBE, from 256 molecule supercells, sampled every 20ps from a $140 \mathrm{ps}$ TIP4P, $N V T$ molecular dynamics trajectory at T=300 K, $\rho=0.996 \mathrm{~g} / \mathrm{cm}^{3}$, with a time averaged EDOS from an uncorrelated TIP4P trajectory using a 32 molecule supercell, computed using $8 \mathrm{k}$-points in the first Brillouin zone. The EDOS are broadened using Lorentzians with a full width at half maximum of $0.05 \mathrm{eV}$. 
ture of homogeneous systems is, perhaps, influenced most by short-range atomic structure, up to second-nearestneighbor interactions. Therefore, we used small, 32 molecule supercells and increased k-point sampling to approximate the electronic structure of larger disordered structures. Such comparisons are consistent when the kpoint density is the same ${ }^{53,54}$ This guarantees that the electronic degrees of freedom encompass the same volume in real-space, the difference being that the system with larger k-point density possesses more structural order, and so is a poorer approximation to the disorderd liquid.

In Fig. 8 we present the EDOS computed using DFT/PBE for large, 256 water molecule supercells. These eight uncorrelated configurations of liquid water are taken from another independent TIP4P trajectory, prepared as outlined in Section V] These EDOS are computed using the $\Gamma$-point approximation and compared with an EDOS representative of a 32 molecule liquid water supercell using $8 \mathrm{k}$-points. This EDOS for the smaller system is actually the average of the 8-kpoint EDOS over the 180 ps presented in Fig. 7. We see that the essential features of the EDOS of the larger system are well reproduced by the smaller system with an equivalent k-point density. In fact the agreement is remarkable considering that there is no correlation between these structures and that the data spans $140 \mathrm{ps}$.

The implications of this agreement are that smaller systems with increased k-point density can accurately represent the electronic structure of larger liquid water supercells. Furthermore, since we require at least $64 \mathrm{k}$ points to converge the EDOS of the 32 molecule configurations (Fig. 7), it is clear that the 256 molecule, $\Gamma$-point calculations are still not converged. The computational expense of generating DFT/GGA electronic structures for such large systems prohibits the comparison with supercells containing 2048 water molecules. Fortunately, it seems that such exhorbitant calculations are unnecessary for accurate prediction of (at least) the DFT electronic stucture of liquid water.

We note also that the qualitative features of the Kohn-Sham eigenstate at the conduction band minimum (Fig. 9) of these very large, 256 molecule supercells are the same as those of the LUMO computed in the smaller $\Gamma$-point calculations and in previous work.

\section{CONSEQUENCES FOR SOLVATION AND SPECTROSCOPY}

At least for the electronic ground state of molecular systems, our calculations show that $\Gamma$-point sampling is sufficient for convergence of the DFT electronic structure. However, care should be taken to verify that the dispersion in such systems is in fact minimal in order to justify this approximation. In particular, simulations involving phase transitions from disordered to ordered phases or from dilute to concentrated phases may exhibit different

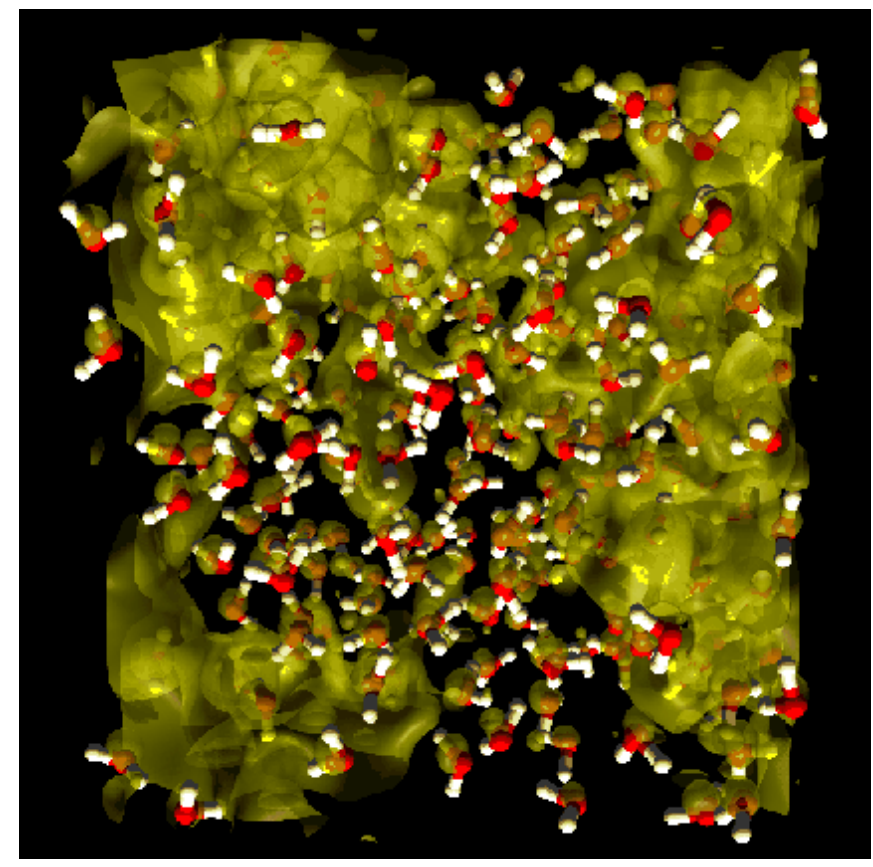

FIG. 9: An isosurface (gold) of the probability density of the Kohn-Sham eigenstate at the conduction band minimum of a 256 molecule supercell of liquid water extracted from a TIP4P trajectory. Water molecules indicated as red (oxygen) and white (hydrogen) ball-and-stick models.

degrees of dispersion in the occupied EDOS, and the minimum k-point sampling required for the more dispersive phase should be adopted.

The large degree of dispersion in the lowest conduction band of liquid water (Fig. 6) may have consequences in the simulation of a hydrated, excess electron. ${ }^{29}$ In the limit of large system size, there should be a continuum of states at the conduction band minimum, allowing for the possibility of "intraband" transitions mediated by finite temperature. It is not clear what impact such transitions may have on the dynamics of such a system and the time scale for localization of the solvated electron.

Of course, all of our work is limited by the accuracy of DFT, and, for excited electronic states, DFT has wellrecognized limitations. This has been explicitly demonstrated for ice $\mathrm{I} h$ in a recent publication 48 However, many-body approaches, such as $\mathrm{GW}, 55$ which allow for improvements in the description of the spectrum of excitations, rely on the use of Kohn-Sham eigenstates as a starting point. Therefore, issues concerning the supercell size and k-point sampling are also relevant for these calculations. In particular, if the size of supercell can be reduced in favor of increased k-point sampling, this will be extremely advantageous for these computationally expensive methodologies.

DFT estimates of the optical absorption spectrum of liquid water will also be sensitive to the system size. We illustrate this in Fig. 10, where we compare the joint density of states (JDOS) of water for various k-point sam- 


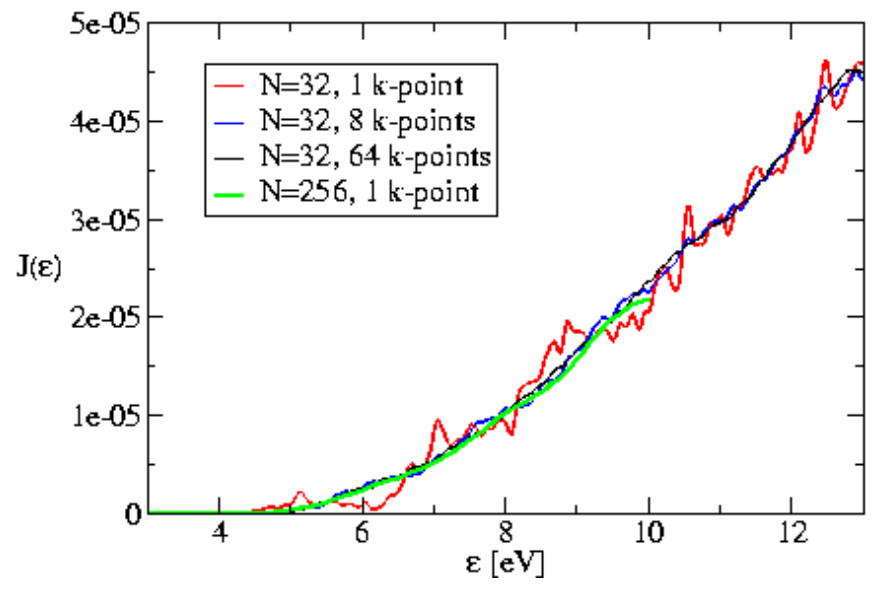

FIG. 10: Convergence of the joint density of states (JDOS) of liquid water (arbitrary units): for a 32 molecule supercell with 1 k-point (red); 8 k-points (blue); 64 k-points (black); and for a 256 molecule supercell with $1 \mathrm{k}$-point (green) with data only up to $10 \mathrm{eV}$ excitations due to a limited number of unoccupied states in this calculation. A Gaussian broadening of $0.05 \mathrm{eV}$ has been employed to expand the JDOS associated with each specific transition.

pling schemes and system sizes. The JDOS is a first estimate of the optical absorption spectrum, ignoring the role of symmetry in electronic transitions from valence to conduction band. We compute the JDOS by considering only interband transitions from the valence to the conduction band. We notice that the JDOS of liquid water is less sensitive to k-point sampling than the underlying EDOS (at least for the broadening scheme we have adopted in Fig. (10). This is to be expected given that the JDOS is a convolution of the valence and conduction band EDOS. Furthermore, the same transferability to larger systems is apparent when we compare the JDOS of a 256 molecule liquid water supercell with that of an uncorrelated, 32 molecule supercell using $8 \mathrm{k}$-points. We note that both of these JDOS are qualitatively different from that computed using the smaller supercell within the $\Gamma$-point approximation. The existence of a peak at the absorption onset is one of the expected consequences of poor Brillouin zone sampling.

DFT investigations into the optical properties of molecules and ions in aqueous solution also require particular attention with respect to accurate representations of the electronic structure of water. Let us ignore, for the moment, the strong possibility of different systematic band gap errors for the solute and solvent within DFT, as has been demonstrated using hybrid exchange correlation functionals 56 If there exists a hybridization of solute and solvent states, which would modify the optical properties, this may be inaccurately estimated using DFT under the $\Gamma$-point approximation, particularly if the relevant, optically active solute state mixes with the bottom of the conduction band of water. 32.33 .34 .56 Under the $\Gamma$-point approximation this state can hybridize with only one water state, whereas our analysis indicates the

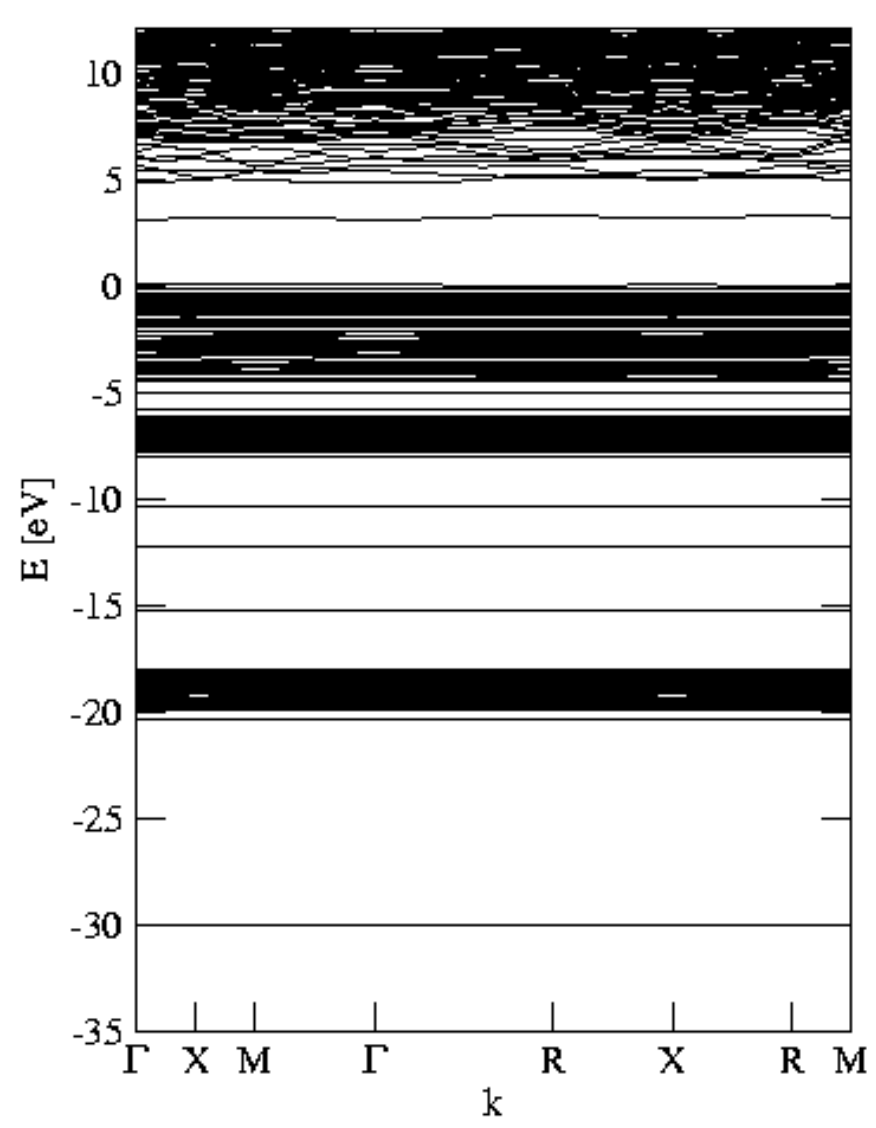

FIG. 11: The "band structure" of a 32 molecule liquid water supercell in the presence of a core (x-ray) excitation from the $1 s$ orbital of one particular oxygen atom modelled using a modified pseudopotential (see text).

presence of a continuum of states in this energy range.

Finally, we demonstate (Fig. 11) the degree of dispersion in the Kohn-Sham eigenstates of a 32 molecule supercell of liquid water in the presence of an x-ray excitation. We model this excitation using a modified pseudopotential which includes a core hole in the $1 s$ level for one particular oxygen atom in the system. The system is effectively ionized and the impact of this perturbation is apparent when the band structure is compared with the ground state (Fig. 6). Localized states are realized, shifted from their respective bands. The conduction band now exhibits states of varying localization, i.e. a subset exhibiting minimal dispersion and the remaining states retaining the typical dispersion associated with the ground state conduction band. Increased k-point sampling beyond the $\Gamma$-point is required to accurately describe all of the conduction band which will be incorporated in spectral calculations for this system.

\section{COMPUTATIONAL EFFICIENCY}

The calculations presented here provide useful guidelines, in general, for the estimation of the electronic prop- 
erties of disordered molecular systems in the condensed phase. While these facts may be well known, we feel that it is useful to reiterate them here as they should be applied to liquid water.

Firstly, it is important to know, for a given supercell size, what amount of k-point sampling is sufficient to describe the occupied subspace of Kohn-Sham eigenstates. This can be easily checked by analysis of the band structure of a representative molecular configuration, or, more consistently, by checking the convergence of the EDOS with respect to k-point sampling. Note that special care is required for the case of possible phase transitions, in small supercells, to states where dispersion is large.

Secondly, as we have shown in this work, knowledge of the degree of dispersion in the occupied subspace is not necessarily transferable to the unoccupied subspace. It may be that a more dense k-point sampling is required for the unoccupied bands. However, given that these states do not influence the electronic charge density of the system, they may be generated non-self-consistently. One may quickly converge the electronic charge density of the system by considering only the occupied states and use a sparse k-point sampling, e.g., just the $\Gamma$-point for a 32 molecule supercell of water. This charge density may be used to generate the common effective potential in a large set of Kohn-Sham equations which are only coupled if they correspond to the same $\mathrm{k}$-point in the Brillouin zone. These equations may be solved using just one matrix diagonalization each, a process that is trivially parallelizable with perfect linear scaling with respect to the number of k-points.

Thirdly, we have shown that the EDOS of a relatively small supercell of water molecules computed with $\mathrm{k}$-points is a very good approximation to the EDOS of a larger system, with an equivalent k-point sampling density. In the case of water, this is a huge saving in computational cost, since increasing the system size eightfold introduces an increase in computational time by a factor of 512 for a typical planewave pseudopotential calculation, which scales at worst as $O\left(N^{3}\right)$, where $N$ is the number of electrons in the system. On the other hand, using $8 \mathrm{k}$-points for the original supercell introduces only an eightfold increase in computational cost, as we have shown. Furthermore, we have also shown that such large calculations: 256 molecules with $\Gamma$-point sampling; are still not converged in the EDOS. So, if we wish to approach a converged result, increased k-point sampling may be the only resort. This necessity does not impose a limit on accuracy in electronic structure calculations for liquid water.

\section{CONCLUSIONS}

In this work, we have provided a clear insight into the electronic structure of liquid water within the context of density functional theory - in particular using the PBE, gradient corrected, exchange correlation functional. We have shown that an accurate representation of the electronic structure is provided by relatively small, 32 molecule supercells of the liquid. By inspection of the convergence of the electronic density of states (EDOS) with respect to $\mathrm{k}$-point sampling of the first Brillouin zone, we have verified that the $\Gamma$-point approximation is adequate for accurate estimation of the occupied EDOS and consequently all ground state electronic properties. However, we find that the $\Gamma$-point approximation is inadequate in providing an accurate description of the unoccupied EDOS of liquid water, which required a $4 \times 4 \times 4$ $\mathrm{k}$-point mesh for convergence. Comparison with larger, uncorrelated, 256 molecule supercells of liquid water indicates that dense $\mathrm{k}$-point sampling for small supercells provides an accurate estimation of the electronic structure of larger supercells which more closely approximate the structural disorder of the liquid. This reveals the possibility of marked savings in computational cost when examining the electronic structure of molecular liquids such as water and, in particular, when attempting to use DFT to estimate the spectroscopic properties of such systems. Work is in progress to calculate both optical and x-ray absorption spectra for liquid water based on the analysis presented here.

\section{Acknowledgments}

We wish to acknowledge T. Ogitsu, G. Cicero, F. Gygi, and A. J. Williamson for useful discussions. We thank N. Marzari for his suggestions regarding PWSCF. This work was performed under the auspices of the U.S. Department of Energy at the University of California/Lawrence Livermore National Laboratory under Contract No. W7405-Eng-48.
1 G. Hura, D. Russo, R. M. Glaeser, T. Head-Gordon, M. Krack, and M. Parrinello, Physical Chemistry Chemical Physics 5, 1981 (2003).

2 A. K. Soper, Chemical Physics 258, 121 (2000).

3 R. Car and M. Parrinello, Physical Review Letters 55, 2471 (1985).

4 P. Hohenberg and W. Kohn, Physical Review 136, B864 (1964).
${ }^{5}$ W. Kohn and L. J. Sham, Physical Review 140, A1133 (1965).

6 J. C. Grossman, E. Schwegler, E. W. Draeger, F. Gygi, and G. Galli, Journal of Chemical Physics 120, 300 (2004).

7 D. Asthagiri, L. R. Pratt, and J. D. Kress, Physical Review E 68, 041505 (2003).

8 A. D. Becke, Physical Review A 38, 3098 (1988).

9 D. C. Langreth and M. J. Mehl, Physical Review B 28, 
1809 (1983).

10 J. P. Perdew, J. A. Chevary, S. H. Vosko, K. A. Jackson, M. R. Pederson, and D. J. Singh, Physical Review B 46, 6671 (1992).

11 E. Schwegler, J. C. Grossman, F. Gygi, and G. Galli, Journal of Chemical Physics 121, 5400 (2004).

12 J. VandeVondele, F. Mohamed, M. Krack, J. Hutter, M. Sprik, and M. Parrinello, Journal of Chemical Physics 122, 014515 (2005)

13 M. Cavalleri, H. Ogasawara, L. Pettersson, and A. Nilsson, Chemical Physics Letters 364, 363 (2002).

14 B. Hetényi, F. D. Angelis, P. Giannozzi, and R. Car, Journal of Chemical Physics 120, 8632 (2004).

15 S. Myneni, Y. Luo, L. A. Näslund, M. Cavalleri, L. Ojamäe, H. Ogasawara, A. Pelmenschikov, P. Wernet, P. Väterlein, C. Heske, et al., Journal of Physics: Condensed Matter 14, L213 (2002).

16 M. Cavalleri, M. Odelius, A. Nilsson, and L. G. M. Pettersson, Journal of Chemical Physics 121, 10065 (2004).

17 P. Wernet, D. Nordlund, U. Bergmann, M. Cavalleri, M. Odelius, H. Ogasawara, L. . A. Näslund, T. K. Hirsch, L. Ojamäe, P. Glatzel, et al., Science 304, 995 (2004).

18 D. Nordlund, H. Ogasawara, P. Wernet, M. Nyberg, M. Odelius, L. Pettersson, and A. Nilsson, Chemical Physics Letters 395, 161 (2004).

19 Y. Q. Cai, H.-K. Mao, P. C. Chow, J. S. Tse, Y. Ma, S. Patchkovskii, J. F. Shu, V. Struzhkin, R. J. Hemley, H. Ishii, et al., Physical Review Letters 94, 025502 (2005).

20 D. T. Bowron, M. H. Krisch, A. C. Barnes, J. L. Finney, A. Kaprolat, and M. Lorenzen, Physical Review B 62, R9223 (2000).

21 U. Bergmann, P. Wernet, P. Glatzel, M. Cavalleri, L. G. M. Pettersson, A. Nilsson, and S. P. Cramer, Physical Review B 66, 092107 (2002).

22 P. Parent, C. Laffon, C. Mangeney, F. Bournel, and M. Tronc, Journal of Chemical Physics 117, 10842 (2002).

23 J. D. Smith, C. D. Cappa, K. R. Wilson, B. M. Messer, R. C. Cohen, and R. J. Saykally, Science 306, 851 (2004).

${ }^{24}$ K. R. Wilson, B. S. Rude, J. Smith, C. Cappa, D. T. Co, R. D. Schaller, M. Larsson, T. Catalano, and R. J. Saykally, Review of Scientific Instruments 75, 725 (2004).

${ }^{25}$ K. Laasonen, M. Sprik, M. Parrinello, and R. Car, Journal of Chemical Physics 99, 9080 (1993).

26 A. D. Becke, Journal of Chemical Physics 96, 2155 (1992).

27 J. P. Perdew, Physical Review B 33, 8822 (1986).

28 M. Boero, K. Terakura, T. Ikeshoji, C. C. Liew, and M. Parrinello, Journal of Chemical Physics 115, 2219 (2001).

${ }^{29}$ M. Boero, M. Parrinello, K. Terakura, T. Ikeshoji, and C. C. Liew, Physical Review Letters 90, 226403 (2003).

${ }^{30}$ F.-Y. Jou and G. R. Freeman, Journal of Physical Chemistry 83, 2383 (1979).

31 M. E. Tuckerman, K. Laasonen, M. Sprik, and M. Parrinello, Journal of Physics: Condensed Matter 6, A93 (1994).

32 L. Bernasconi, M. Sprik, and J. Hutter, Journal of Chemical Physics 119, 12417 (2003).
33 L. Bernasconi, J. Blumberger, M. Sprik, and R. Vuilleumier, Journal of Chemical Physics 121, 11885 (2004).

34 D. Prendergast, J. C. Grossman, A. J. Williamson, J.-L. Fattebert, and G. Galli, Journal of the American Chemical Society 126, 13827 (2004).

${ }^{35}$ F. Gygi, GP 1.24.0: A General Ab Initio Molecular Dynamics Program, Lawrence Livermore National Laboratory, Livermore, CA (2003).

36 X. Gonze, J.-M. Beuken, R. Caracas, F. Detraux, M. Fuchs, G.-M. Rignanese, L. Sindic, M. Verstraete, G. Zerah, F. Jollet, et al., Computational Materials Science 25, 478 (2002).

37 J. P. Perdew, K. Burke, and M. Ernzerhof, Physical Review Letters 77, 3865 (1996).

38 D. R. Hamann, Physical Review B 40, 2980 (1989).

39 A. Bernas, C. Ferradini, and J.-P. Jay-Gerin, Chemical Physics 222, 151 (1997).

40 H. Hayashi, N. Watanabe, Y. Udagawa, and C.-C. Kao, Proceedings of the National Academy of Sciences 97, 6264 (2000).

41 S. Baroni, A. D. Corso, S. de Gironcoli, and P. Giannozzi, URL http: //www . pwscf . org

42 P. Jedlovszky, J. P. Brodholt, F. Bruni, M. A. Ricci, A. K. Soper, and R. Vallauri, Journal of Chemical Physics 108, 8528 (1998).

43 G. P. Parravicini and L. Resca, Physical Review B 8, 3009 (1973).

44 Y.-N. Xu and W. Y. Ching, Physical Review B 50, 17709 (1994).

45 J. Bai, C.-R. Su, R. D. Parra, X. C. Zeng, H. Tanaka, K. Koga, and J.-M. Li, Journal of Chemical Physics 118, 3913 (2003).

46 J. D. Bernal and R. H. Fowler, Journal of Chemical Physics 1, 515 (1933).

47 D. R. Hamann, Physical Review B 55, R10157 (1997).

48 P. H. Hahn, W. G. Schmidt, K. Seino, M. Preuss, F. Bechstedt, and J. Bernholc (2005), accepted for publication in Physical Review Letters.

49 W. L. Jorgensen, J. Chandrasekhar, J. D. Madura, R. W. Impey, and M. L. Klein, Journal of Chemical Physics 79, 926 (1983).

50 E. Sanz, C. Vega, J. L. F. Abascal, and L. MacDowell, Physical Review Letters 92, 255701 (2004).

51 H. J. C. Berendsen, D. van der Spoel, and R. van Drunen, Comp. Phys. Comm. 91, 43 (1995).

52 E. Lindahl, B. Hess, and D. van der Spoel, J. Mol. Mod. 7, 306 (2001).

53 G. Makov and M. C. Payne, Physical Review B 51, 4014 (1995).

54 G. Makov, R. Shah, and M. C. Payne, Physical Review B 53, 15513 (1996).

55 M. S. Hybertsen and S. G. Louie, Physical Review B 34, 5390 (1986).

${ }^{56}$ L. Bernasconi, M. Sprik, and J. Hutter, Chemical Physics Letters 394, 141 (2004). 